\title{
Role of Water in Aging of Human Butyrylcholinesterase Inhibited by Echothiophate: The Crystal Structure Suggests Two Alternative Mechanisms of Aging ${ }^{\dagger, *}$
}

\author{
Florian Nachon, ${ }^{*, \S, \|}$ Oluwatoyin A. Asojo, ${ }^{\S}$ Gloria E. O. Borgstahl, ${ }^{\S}$ Patrick Masson," and Oksana Lockridge ${ }^{\S}$ \\ The Eppley Institute for Research in Cancer and Allied Diseases, University of Nebraska Medical Center, \\ Omaha, Nebraska 68198-6805, and Unité d'enzymologie, Département de Toxicologie, Centre de Recherches du Service de \\ Santé des Armées, 24 avenue des Maquis du Grésivaudan BP87, 38702 La Tronche Cedex, France
}

Received August 14, 2004; Revised Manuscript Received November 12, 2004

\begin{abstract}
Organophosphorus poisons (OP) bind covalently to the active-site serine of cholinesterases. The inhibited enzyme can usually be reactivated with powerful nucleophiles such as oximes. However, the covalently bound $\mathrm{OP}$ can undergo a suicide reaction (termed aging) yielding nonreactivatable enzyme. In human butyrylcholinesterase (hBChE), aging involves the residues His438 and Glu197 that are proximal to the active-site serine (Ser198). The mechanism of aging is known in detail for the nerve gases soman, sarin, and tabun as well as the pesticide metabolite isomalathion. Aging of soman- and sarin-inhibited acetylcholinesterase occurs by $\mathrm{C}-\mathrm{O}$ bond cleavage, whereas that of tabun- and isomalathion-inhibited acetylcholinesterase occurs by $\mathrm{P}-\mathrm{N}$ and $\mathrm{P}-\mathrm{S}$ bond cleavage, respectively. In this work, the crystal structures of $\mathrm{hBChE}$ inhibited by the ophthalmic reagents echothiophate (nonaged and aged) and diisopropylfluorophosphate (aged) were solved and refined to $2.1,2.25$, and $2.2 \AA$ resolution, respectively. No appreciable shift in the position of the catalytic triad histidine was observed between the aged and nonaged conjugates of hBChE. This absence of shift contrasts with the aged and nonaged crystal structures of Torpedo californica acetylcholinesterase inhibited by the nerve agent VX. The nonaged hBChE structure shows one water molecule interacting with Glu197 and the catalytic triad histidine (His438). Interestingly, this water molecule is ideally positioned to promote aging by two mechanisms: breaking either a $\mathrm{C}-\mathrm{O}$ bond or a $\mathrm{P}-\mathrm{O}$ bond. Pesticides and certain stereoisomers of nerve agents are expected to undergo aging by breaking the $\mathrm{P}-\mathrm{O}$ bond.
\end{abstract}

Organophosphorus compounds $(\mathrm{OP})^{1}$ are widely used as pesticides, plasticizers, pharmaceuticals, in hydraulic fluid, and in jet engine fuel. Some are feared chemical warfare agents. Acute toxicity from exposure to organophosphorus agents is due to inhibition of acetylcholinesterase (AChE; EC 3.1.1.7). Butyrylcholinesterase (BChE; EC 3.1.1.8), a closely related serine hydrolase found in vertebrates and sharing about 55\% sequence identity with $\mathrm{AChE}$, is also inhibited. But $\mathrm{BChE}$ inhibition is thought to serve a protective role, by scavenging OP molecules. Plasma BChE scavenges

† Supported by U.S. Army Medical Research and Materiel Command, Grant DAMD17-01-2-0036 (to O.L.); UNMC Eppley Cancer Center Support, Grant P30CA36727-19; Contract 00-2-032-0-00 from the Office of the Defense Cooperation Attache, République Française, Washington, DC, part of DGA 99CO-029/PEA (to P.M.); DGA/DSP/ STTC-PEA 010807 (to P.M. and O.L.); and the Nebraska Tobacco Settlement Biomedical Research Development Fund (to G.B.).

$\star$ Protein Data Bank entry codes are 1XLW for nonaged echothiophateinhibited hBChE, 1XLV for aged echothiophate-inhibited hBChE, and 1XLU for aged DFP-inhibited hBChE.

* To whom correspondence should be addressed: Telephone $(+33)$ 47663 9765; fax (+33) 47663 6962; e-mail florian@ nachon.net.

$\S$ University of Nebraska Medical Center.

"Centre de Recherches du Service de Santé des Armées.

${ }^{1}$ Abbreviations: OP, organophosphate; AChE, acetylcholinesterase; BChE, butyrylcholinesterase; DFP, diisopropyl fluorophosphate; DP, diethylphosphoryl; VX, $O$-ethyl-S-[2-[bis(1-methylethyl)amino]ethyl]methylphosphonothioate; PDB, Protein Data Bank.
OPs, thus preventing inhibition of neuromuscular and neuronal AChEs. Monkeys pretreated with human $\mathrm{BChE}(\mathrm{hBChE})$ can be fully protected against OP toxicity, for example, up to $5 \mathrm{LD}_{50}$ of soman $(1-3)$.

Aging is defined as a time-dependent process leading to nonreactivatable enzyme. For example, soman-inhibited acetylcholinesterase ages by dealkylation (departure of the pinacolyl side chain) of the OP moiety (4). Many OP react faster with $\mathrm{BChEs}$ than with AChEs (5-7), and many $\mathrm{OP}-$ $\mathrm{BChE}$ conjugates age faster than $\mathrm{OP}-\mathrm{AChE}$ conjugates (Table 1). The goal of this study was to understand the particularities of the BChE aging mechanism. For this purpose we solved the crystal structures of OP-inhibited human $\mathrm{BChE}$ before and after aging.

We focused on hBChE inhibited by the ophthalmic agents echothiophate iodide and diisopropylfluorophosphate (DFP) (see structures in Figure 1). Echothiophate was used because (a) the diethylphosphoryl conjugate ages slowly, with a halflife of $12 \mathrm{~h}$ at $\mathrm{pH} 7.0(8)$, allowing isolation of the first nonaged conjugate of $\mathrm{hBChE}$ to date, as well as the aged structure; (b) the aged derivative is close in size to butyrate, making the aged structure analogous to the deacylation tetrahedral intermediate during hydrolysis of butyrylcholine; (c) echothiophate iodide is soluble and stable in aqueous solvent and therefore readily soaks into a crystal; (d) 


\begin{tabular}{|c|c|c|c|c|c|c|}
\hline Enzyme & OP & $\begin{array}{l}\text { Substituent } \\
\qquad \mathrm{R}_{1}, \mathrm{R}_{2}\end{array}$ & $\begin{array}{c}\text { Aging } \\
\text { half-life }\end{array}$ & $\mathrm{pH}$ & $\begin{array}{c}\mathrm{T} \\
\text { in }^{\circ} \mathrm{C}\end{array}$ & Reference \\
\hline \multicolumn{7}{|l|}{$\mathrm{hBChE}$} \\
\hline & Paraoxon & $\mathrm{EtO}, \mathrm{EtO}$ & $11.6 \mathrm{~h}$ & 8 & 20 & (8) \\
\hline & Triazophos & $\mathrm{EtO}, \mathrm{EtO}$ & $12.6 \mathrm{~h}$ & 7.4 & 37 & $(31)$ \\
\hline & DFP & iPrO, iPrO & $1 \mathrm{~h}$ & 8 & 25 & (32) \\
\hline & Cyclosarin & $\mathrm{Me}$ & $2.2 \mathrm{~h}$ & 7.4 & 37 & (33) \\
\hline & Soman & & & & & \\
\hline & (racemic) & , Me & $9 \mathrm{~min}$ & 8 & 25 & (32) \\
\hline & Soman & & & & & \\
\hline & $\mathrm{PsCs}$ & , Me & $3.9 \mathrm{~min}$ & 8 & 25 & (27) \\
\hline \multicolumn{7}{|l|}{ hAChE } \\
\hline & Paraoxon & $\mathrm{EtO}, \mathrm{EtO}$ & $41 \mathrm{~h}$ & & 37 & (34) \\
\hline & Ethyldichlorvos & $\mathrm{EtO}, \mathrm{EtO}$ & $58 \mathrm{~h}$ & 7.4 & 37 & (35) \\
\hline & DFP & iPrO, iPrO & $4.6 \mathrm{~h}$ & & 37 & (34) \\
\hline & Cyclosarin & , Me & $8.7 \mathrm{~h}$ & 7.4 & 37 & (33) \\
\hline & Cyclosarin & Me & $4.6 \mathrm{~h}$ & $7.1-7.3$ & 25 & $(36)$ \\
\hline & Soman &, $\mathrm{Me}$ & $6.3 \mathrm{~min}$ & 8 & 27 & $(37)$ \\
\hline
\end{tabular}

echothiophate is not volatile, making it relatively safe in a laboratory setting; (e) echothiophate is of clinical interest because it has been used as an anti-glaucoma drug; (f) echothiophate is positively charged and does not cross the blood-brain barrier; and last, ( $\mathrm{g}$ ) echothiophate is a prototype of the VX nerve agent family. DFP was used to allow comparison of DFP-phosphorylated hBChE with the DFPinhibited Torpedo californica AChE (TcAChE) structure (9).

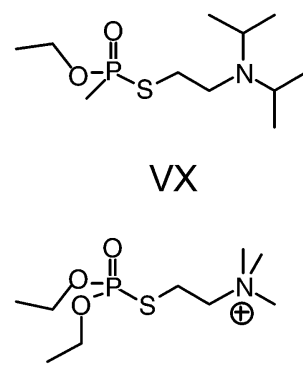

Echothiophate

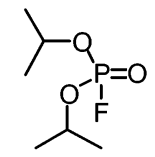

DFP

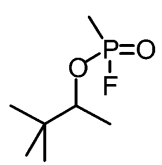

Soman
FIGURE 1: Chemical structures of VX, DFP, echothiophate, and soman.

Of particular interest was to compare the OP-hBChE and OP-TcAChE structures in order to understand why they age at different rates. Millard et al. (10) reported the crystal structure of nonaged VX-inhibited TcAChE (PDB entry
1VXO). They found that the catalytic His $440^{2}$ shifted $4.5 \AA$ away from its triad partner Glu327 to interact with Glu199. They suggested disruption of the catalytic triad could explain the slow spontaneous reactivation of the $\mathrm{VX}-T c \mathrm{AChE}$ conjugate. In the aged structure, the imidazole ring of His440 had returned to its idle position. It was of interest to determine whether the catalytic His of $\mathrm{hBChE}$ displayed a similar mobility during aging. Aged TcAChE structures, resulting from reaction with soman, sarin, DFP, or VX, are essentially identical and provide an explanation for the resistance to reactivation of the aged OP conjugates $(9,10)$. The present work asked whether the mechanism of aging was equivalent in phosphylated $\mathrm{AChE}$ and $\mathrm{BChE}$.

\section{MATERIALS AND METHODS}

Crystals of Nonaged Echothiophate-Inhibited hBChE. The recombinant hBChE was a truncated monomer containing residues $1-529$ (11). The tetramerization domain at the carboxy terminus was deleted. The carbohydrate content was reduced by site-directed mutagenesis from 9 to 6 glycans (11). The recombinant hBChE gene was expressed in Chinese hamster ovary $(\mathrm{CHO})$ cells. The enzyme secreted into serumfree culture medium was purified by affinity and ionexchange chromatography and crystallized as described (11). The mother liquor was 0.1 M MES buffer, pH 6.5, with 2.1

${ }^{2} T c \mathrm{AChE}$ 's amino acid numbering corresponds to hBChE's amino acid numbering +2 . 


\begin{tabular}{|c|c|c|c|}
\hline & MIP-BChE (DFP aged) & DEP-BChE (echothiophate) & EFS-BChE (echothiophate aged) \\
\hline \multicolumn{4}{|c|}{ Data } \\
\hline space group & I422 & I422 & I422 \\
\hline unit cell axes, $a=b, c(\AA)$ & $154.49,127.29$ & $154.46,127.01$ & $154.59,126.70$ \\
\hline no. of reflections & 294819 & 402015 & 247056 \\
\hline unique reflections & 37851 & 43695 & 31,580 \\
\hline resolution $(\AA)$ & $50-2.20(2.20-2.28)$ & $50-2.10(2.18-2.10)$ & $50-2.25(2.33-2.25)$ \\
\hline completeness (\%) & $96.3(100.0)$ & $97.3(98.2)$ & $86.1(91.1)$ \\
\hline$R_{\text {merge }}^{a}(\%)$ & $6.3(40.1)$ & $5.8(46.2)$ & $5.1(43.9)$ \\
\hline$I / \sigma(\mathrm{I})$ & $27.9(4.6)$ & $29.0(5.5)$ & $32.2(3.3)$ \\
\hline redundancy & $7.8(7.7)$ & $9.2(9.0)$ & $7.8(6.3)$ \\
\hline \multicolumn{4}{|c|}{ Refinement Statistics } \\
\hline$R$-factor ${ }^{b}\left(R\right.$-free $\left.{ }^{c}\right)$ & $19.3(24.2)$ & $18.4(22.3)$ & $18.3(24.6)$ \\
\hline \multicolumn{4}{|l|}{ no. of atoms } \\
\hline protein & 4273 & 4225 & 4285 \\
\hline solvent & 255 & 290 & 220 \\
\hline others & 158 & 192 & 172 \\
\hline mean $B$-factor $\left(\AA^{2}\right)$ & 45.8 & 42.3 & 48.3 \\
\hline \multicolumn{4}{|l|}{ RMS from ideality } \\
\hline bond length $(\AA)$ & 0.017 & 0.018 & 0.018 \\
\hline angles (deg) & 1.600 & 1.714 & 1.744 \\
\hline $\operatorname{chiral}\left(\AA^{3}\right)$ & 0.109 & 0.127 & 0.115 \\
\hline
\end{tabular}

${ }^{a} R_{\text {merge }}=(\Sigma|I-\langle I\rangle|) / \Sigma I$, where $I$ is the observed intensity and $\langle I\rangle$ is the average intensity obtained from multiple observations of symmetryrelated reflections after rejections. ${ }^{b} R$-factor $=\Sigma\left|F_{\mathrm{o}}-\right| F_{\mathrm{c}}|| \Sigma\left|F_{\mathrm{o}}\right|$, where $F_{\mathrm{o}}$ are observed and $F_{\mathrm{c}}$ are calculated structure factors. ${ }^{c} R$-free set uses $5 \%$ of randomly chosen reflections defined in Brunger et al. (38).

$\mathrm{M}\left(\mathrm{NH}_{4}\right)_{2} \mathrm{SO}_{4}$. The echothiophate iodide (Wyeth-Ayerst, Rouses Point, NY) stock solution was $50 \mathrm{mM}$ in water. The echothiophate $-\mathrm{hBChE}$ conjugate was obtained by soaking crystals for $1 \mathrm{~h}$ in $0.1 \mathrm{M}$ MES buffer, $\mathrm{pH} 6.5$, with $2.4 \mathrm{M}$ $\left(\mathrm{NH}_{4}\right)_{2} \mathrm{SO}_{4}$ containing $1 \mathrm{mM}$ echothiophate iodide. Data were collected right after the incubation to avoid aging ( $t_{1 / 2}$ aging $\approx 12 \mathrm{~h}$ at $\mathrm{pH} 7.0,25^{\circ} \mathrm{C}$ ).

Crystallization of DFP and Echothiophate-Aged hBChE. The purified enzyme $(6.6 \mathrm{mg} / \mathrm{mL})$ was inhibited in the presence of $0.5 \mathrm{mM}$ DFP (Sigma) in $10 \mathrm{mM}$ MES buffer, $\mathrm{pH}$ 6.5. The reaction mixture was incubated for 1 day at 4 ${ }^{\circ} \mathrm{C}$, allowing enough time for completion of the aging reaction $\left(t_{1 / 2}\right.$ aging $\approx 1 \mathrm{~h}$ at $\left.\mathrm{pH} 7.0,25^{\circ} \mathrm{C}\right)$. The inhibited enzyme was crystallized by the hanging drop method as described (12). Crystals grew to $0.5 \times 0.5 \times 0.3 \mathrm{~mm}$ in one week at $20^{\circ} \mathrm{C}$. The echothiophate-aged $\mathrm{hBChE}$ crystals were produced following a similar process after the enzyme was inhibited in the presence of $1 \mathrm{mM}$ echothiophate iodide in $10 \mathrm{mM}$ MES buffer, $\mathrm{pH}$ 6.5. The length of time between the phosphorylation step and the data collection was sufficiently long ( $>1$ week) to achieve completion of the aging reaction.

$X$-ray Data Collection and Structure Solution. Data were collected at the University of Nebraska Medical Center Structural Biology Laboratory. The crystals were washed with a cryoprotectant solution [0.1 M MES buffer with 2.4 $\mathrm{M}\left(\mathrm{NH}_{4}\right)_{2} \mathrm{SO}_{4}$ containing $18 \%$ glycerol] and then flash-cooled at $-160{ }^{\circ} \mathrm{C}$ in a nitrogen stream provided by an X-Stream 2000 system (Rigaku MSC). The X-ray diffraction equipment consisted of a Rigaku rotating anode FR-E SuperBright X-ray generator, Osmic mirrors for focusing, and a RAXIS-IV ++ detector.

All data sets were processed with HKL2000 (13). The structures were solved by use of the CCP4 suite (14). An initial solution model was determined by molecular replacement with MolRep (15) starting from the recombinant BChE structure (PDB entry 1P0I) from which all ligands (butyrate, glycerol, ions) and glycan chains had been removed. For all diffraction data sets, the model was refined by the following strategy: an initial rigid-body refinement with REFMAC5 (16), followed by iterative cycles of model building, with PyMol (17) and $\mathrm{O}$ (18), and restrained refinement with REFMAC5.

\section{RESULTS}

$X$-ray Crystallography. Data were collected from tetragonal crystals of space group $I 422$ and refined to $2.20 \AA$ (DFP-phosphorylated enzyme), $2.10 \AA$ (nonaged echothiophate$\mathrm{BChE}$ conjugate), and $2.25 \AA$ (aged echothiophate $-\mathrm{BChE}$ conjugate). Data and refinement statistics are shown in Table 2. In the original structure (PDB entry 1P0I) the catalytic serine was within $2.16 \AA$ of a butyrate molecule. The butyrate was removed in the starting model used for the molecular replacement. Therefore the peak of positive density within covalent bond distance of the $\mathrm{O} \gamma$ of the catalytic serine, observed in the omit electron density maps of the three OP structures, confirms the presence of the covalently bound inhibitor.

A peak of positive density was found close to Cys66 in the three structures. This density was refined as a sulfur atom covalently bound to Cys66 ( $S$-mercaptocysteine). While this particular modification of residue 66 is present in the enzyme preparation we used in this work, it was clearly absent in the previously solved structures of hBChE (12).

Induced Fit of the Acyl Loop. One side of the active-site gorge is delimited by a loop, called the acyl-loop, because it extends from residue 276 at the gorge entrance to residue 289 in the acyl-binding pocket. The acyl-binding pocket of $\mathrm{hBChE}$ is defined by side chains of residues Leu286, Val288, and Trp231. The function of this pocket is to bind the acyl moiety of carboxyl ester substrates. This pocket also binds one isopropoxy group of DFP in the DP conjugate and the methyl group of soman-phosphonylated BChE (Figure 2A). The conformation of the acyl-loop in the DFP-phosphorylated 


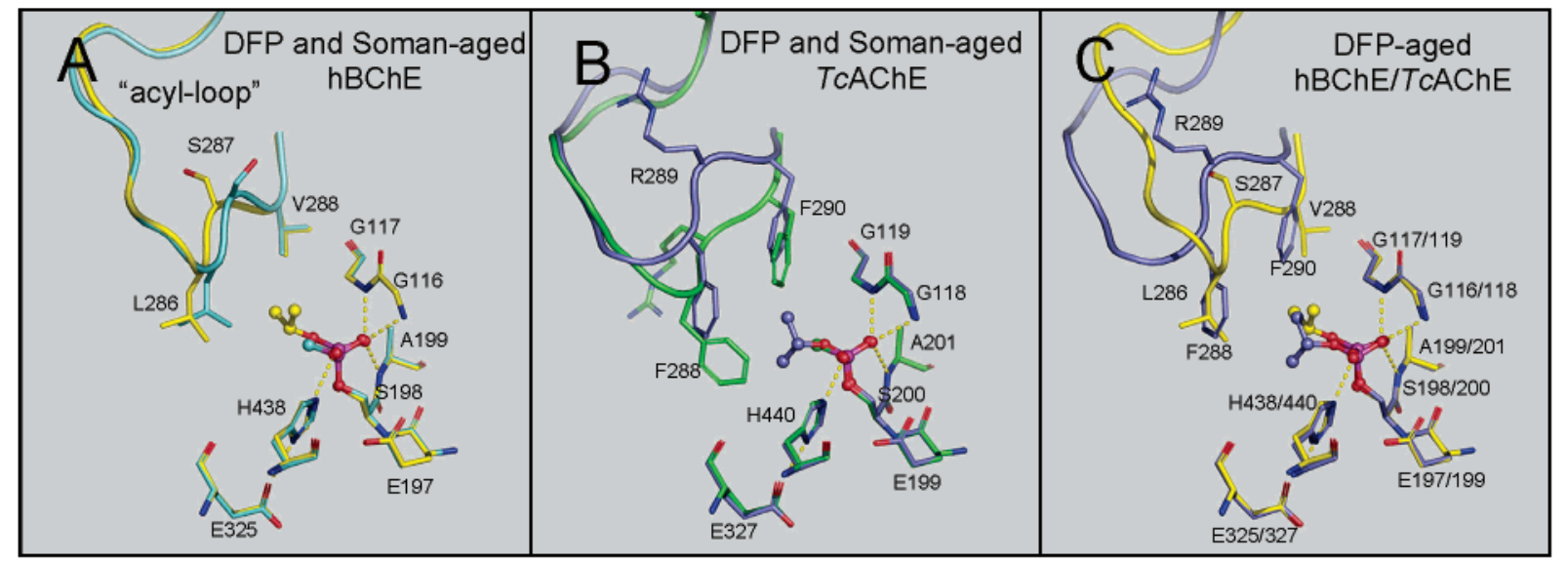

FIGURE 2: Superposition of the active-site structures of aged hBChE and aged TcAChE emphasizing the differences in the "acyl-loop" conformations. (A) Superposition of aged DFP-inhibited hBChE, carbon atoms in yellow, and aged soman-inhibited hBChE (PDB entry 1P0Q) (12), carbon atoms in light blue. (B) Aged DFP-inhibited TcAChE (PDB entry 2DFP) (9), carbon atoms in slate, and aged Somaninhibited TcAChE (PDB entry 1SOM) (9), carbon atoms in green. (C) Aged DFP-inhibited hBChE, carbon atoms in yellow, and aged DFP-inhibited $T c$ AChE, carbon atoms in slate. The backbone of the acyl-loops is represented as a thick wire. Key residues are represented as sticks with nitrogen atoms in blue and oxygen atoms in red. The phosphoryl- (DFP) and phosphonyl- (soman) heads are represented as ball-and-sticks with the phosphorus atoms in magenta. Hydrogen bonds are represented by yellow dashes.

enzyme structure is the same as in the butyrate- $\mathrm{hBChE}$ structure (PDB entry 1P0I). However, its conformation is different from the soman-aged hBChE structure. The van der Waals volume of the methylphosphonyl moiety derived from soman inhibition is smaller than the one occupied by the monoisopropylphosphoryl aged moiety resulting from DFP inhibition. The size difference results from moietyinduced fit of the acyl-loop. In the soman-aged structure, Leu286 is shifted inward by $2.0 \AA$, clasping the acyl-binding pocket around the methyl group. The Leu286 shift is accompanied by a flip of the adjacent residue, Ser287.

The acyl-binding pocket of TcAChE is shaped by Trp233 and two additional bulky aromatic residues, Phe288 and Phe290, both much bulkier than their aliphatic counterparts in hBChE, Leu286 and Val288. The acyl-binding pocket of TcAChE is therefore more restrictive than that of $\mathrm{hBChE}$. The restrictive acyl pocket of AChE provides a good fit for the small methyl group of the soman-aged TcAChE structure. However, the pocket is too small to accommodate larger groups, such as the isopropoxy group of the DP moiety, without adjustment. Figure 2B shows the shift in the acylloop backbone reported by Millard et al. (9). The conformational change is accompanied by a flip of Arg289, the residue between Phe288 and Phe290. This is similar to the flip of Ser287, the equivalent residue in hBChE.

As a consequence of the conformational change following inhibition by DFP, the size of the acyl-binding pocket in TcAChE has expanded to become similar in size to the acylbinding pocket in $\mathrm{hBChE}$, even though the conformations of the acyl-loop backbones are very different $(2.74 \AA \mathrm{rms}$ deviation). This is shown in Figure 2C. With the exception of the acyl-loop, the active-site residues have the same positions in DFP-aged $\mathrm{hBChE}$ and AChE. The isopropyl groups do not superimpose in $T c \mathrm{AChE}$ and $\mathrm{hBChE}$, reflecting the specificity of the interactions with the different side chains of the two pockets.

No Change in the Position of His438 in EchothiophateInhibited, Nonaged hBChE. The crystal structure of the nonaged, echothiophate-inhibited $\mathrm{hBChE}$ shows the phosphorus atom covalently bound to the $\mathrm{O} \gamma$ of the catalytic
Ser198. One oxygen of the phosphate fits in the oxyanion hole, strongly hydrogen-bonded to the backbone $\mathrm{NH}$ of Gly116, Gly117, and Ala199 (Figure 3A). The distances are $2.81 \AA$ to Gly116, $2.75 \AA$ to Gly117, and $2.94 \AA$ to Ala199. One ethoxy group fills the acyl-binding pocket. The second ethoxy group points toward the mouth of the gorge. The oxygen atom of the second ethoxy group is at hydrogenbonding distance to $\mathrm{N} \in 2$ of His438 (3.32 $\AA$ ).

The catalytic triad of hBChE consists of Ser198, His438, and Glu325. These residues are interconnected by hydrogen bonds in hBChE when Ser198is not interacting with a ligand. This situation is observed for one of the alternate conformations of the catalytic serine in the choline-hBChE complex (PDB entry 1P0M). However, the Ser198-His438 connection is disrupted in the diethylphosphoryl conjugate of hBChE (Figure 3A). Though the $\mathrm{N} \in 2$ of His438 is at hydrogen-bonding distance to $\mathrm{O} \gamma$ of $\operatorname{Ser} 198$ (3.22 $\AA$ ), the angle is about $80^{\circ}$, a value unfavorable for making a $\mathrm{H}$-bond. This was also observed in the butyrate-hBChE complex (PDB entry 1P0I). By contrast, His438 retains its connection to Glu325 in the diethylphosphoryl conjugate. The interaction with Glu325 maintains the histidine in the same position in all hBChE crystal structures observed to date. The disruption of the H-bond between $\mathrm{O} \gamma$ of the serine and $\mathrm{N} \epsilon 2$ of His 438 when a ligand is bound to the serine does not mean that the catalytic residues adopt an improper position for catalytic function. Actually, it is necessary that the relative positions of the serine and histidine side chains adjust during the catalytic cycle. This allows efficient proton transfer between the serine, the leaving alcohol product, and the water molecule that hydrolyzes the acyl-enzyme.

A Possible Second Mechanism of Aging. Another important feature of the diethylphosphoryl-hBChE conjugate (Figure $3 \mathrm{~A})$ is the location of water molecule 101. Water 101 is within interaction distance to $\mathrm{N} \epsilon 2$ of His438 (2.90 $\AA$ ) and the carboxyl group of Glu197 (2.82 ̊). Therefore, water 101 serves as a relay for Glu197 to stabilize the positive charge on protonated His438. Because of its interaction with Glu197, Water 101 is activated. This water molecule is ideally positioned to catalyze two mechanisms of aging. 


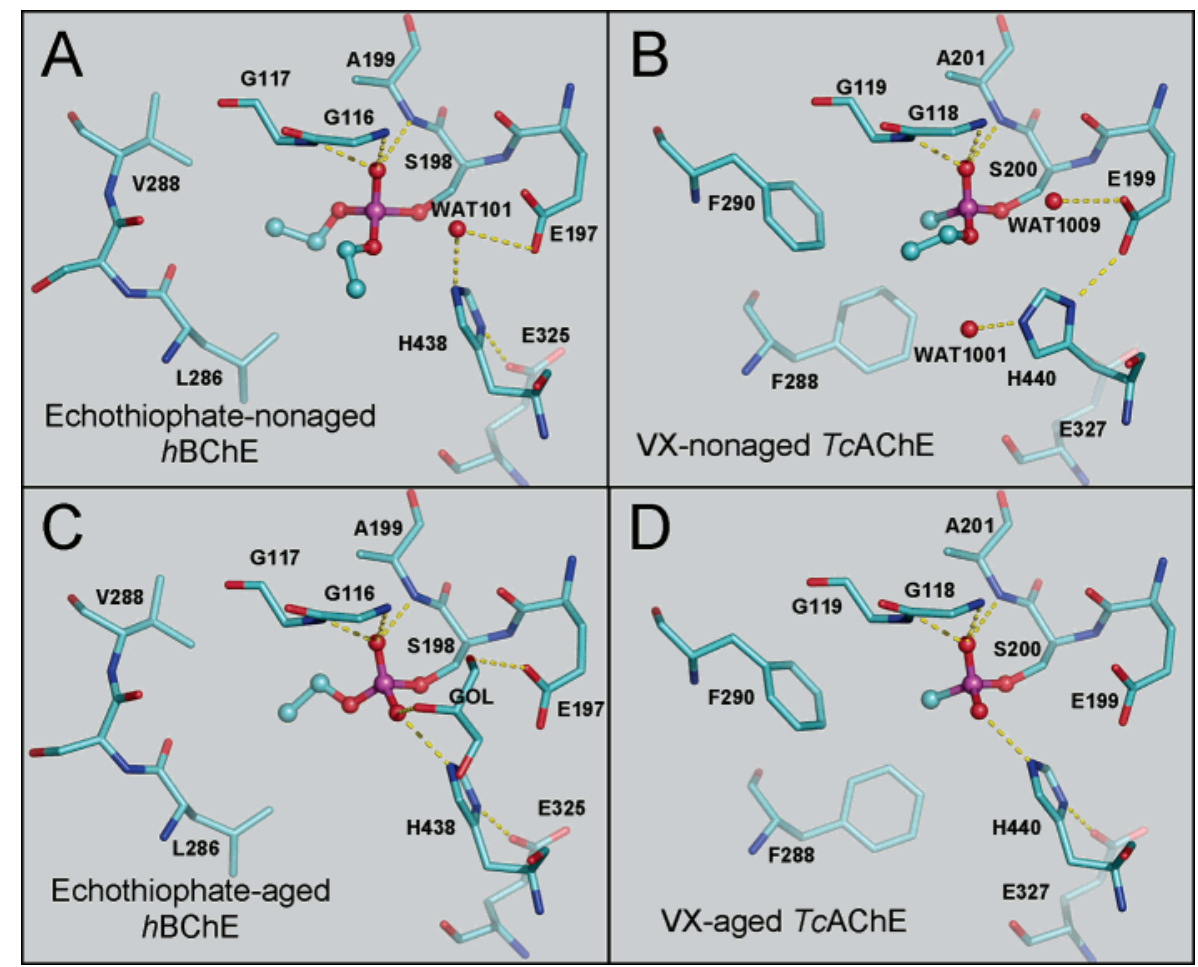

FIGURE 3: Active-site structures of $\mathrm{hBChE}$ and $T c \mathrm{AChE}$ before and after aging. (A) hBChE inhibited by echothiophate yields a diethylphosphoryl conjugate (nonaged). (B) Diethylphosphoryl-hBChE aged to a monoethylphosphoryl conjugate. (C) TcAChE inhibited by VX yields the $O$-ethylmethylphosphonyl conjugate (nonaged; PDB entry $1 \mathrm{VXR}$ ) (10). (D) TcAChE-conjugate aged into a methylphosphonyl conjugate (PDB entry 1VXO) (9). Key active-site residues and glycerol (GOL) are represented as sticks, the OP as ball-and-sticks, and water molecules as red spheres. Water 101 in $\mathrm{BChE}$ is positioned to carry out aging by either $\mathrm{C}-\mathrm{O}$ or $\mathrm{P}-\mathrm{O}$ bond breakage.

In the first aging mechanism the $\mathrm{C}-\mathrm{O}$ bond of the ethoxy group is broken. This is the accepted mechanism of aging of acetylcholinesterase for soman $(4,19)$ and sarin $(20)$. The positively charged imidazolium ion stabilizes the developing negative charge on the $\mathrm{C}-\mathrm{O}^{\delta-}$ oxygen. Water 101 is at a good distance to attack the carbocationic center that appears on the $\mathrm{C}^{\delta+}-\mathrm{O}$ carbon.

In the second aging mechanism the $\mathrm{P}-\mathrm{O}$ bond is broken. Water 101 is ideally positioned to attack the electrophilic phosphorus atom to form a bipyramidal intermediate. The leaving group has to be on the opposite side of the attacking water molecule (in-line inversion). Therefore, the leaving group is the ethoxy group that fills the acyl-binding pocket. This second mechanism is a novel result from this work.

The diethylphosphoryl $-\mathrm{hBChE}$ conjugate has the ability to age by both mechanisms. Though the energy needed to break $\mathrm{C}-\mathrm{O}$ and $\mathrm{P}-\mathrm{O}$ bonds is of the same order, that is, about $360 \mathrm{~kJ} \cdot \mathrm{mol}^{-1}$, local geometry, polarity, and dielectric constraints may dramatically alter the activation energy needed for covalent bond breakage. Therefore, it is risky to predict which mechanism predominates.

Comparison of Echothiophate-Inhibited Nonaged hBChE to VX-Inhibited Nonaged TcAChE. The structure of VXinhibited $T c \mathrm{AChE}$ before aging is shown in Figure 3B (PDB entry 1VXR) (10). The similarities with the $\mathrm{hBChE}$ structure in Figure $3 \mathrm{~A}$ are the following. The oxygen of the phosphonate is locked in the oxyanion hole, the methyl group is in the acyl pocket, and the ethoxy group points toward the mouth of the gorge. Water 1009 in TcAChE is in a position equivalent to that of water $101 \mathrm{in} \mathrm{hBChE.} \mathrm{However,} \mathrm{water}$ 1009 is not within hydrogen-bond distance of His 440 because His440 has shifted its position. This contrasts with $\mathrm{hBChE}$, whose catalytic histidine does not shift position after inhibition by OP. Although the position of Water 1009 is compatible with an attack on the phosphorus atom, the reaction cannot take place. The reason is that in the VXinhibited TcAChE, the group on the opposite side of the bipyramidal intermediate is a methyl group directly bound to the phosphorus atom, that is, not a leaving group. Thus, the second aging mechanism involving breaking of a $\mathrm{P}-\mathrm{O}$ bond is not possible for VX-inhibited TcAChE. The same is probably true for dealkylation of sarin- and soman-inhibited TcAChE.

The connection between glutamate Glu327 of the catalytic triad and His440 is disrupted in TcAChE but not in $\mathrm{hBChE}$ (compare Figure 3 panels A and B). The consequence is that the imidazolium ion does not directly stabilize the negative charge on the oxygen of the $\mathrm{C}-\mathrm{O}$ bond. Millard et al. (10) concluded this explains the slow aging of VX-inhibited TcAChE.

However, Water 1001 in Figure 3B is part of a hydrogenbonded network, including Glu199 and His440. Water 1001 is $3.58 \AA$ away from the primary carbon of the ethoxy group. This water molecule is activated and may stabilize the formation of the developing carbocation. An equivalent water molecule is not present in echothiophate-phosphorylated $\mathrm{hBChE}$ (Figure 3A).

Aged $h B C h E$ and TcAChE. The dealkylation leads to a new negative charge on one of the oxygens of the phosphyl group. This has major consequences. The presence of this negative charge decreases the electrophilicity of the phosphorus atom so that nucleophiles, like oximes, are unable to displace the phosphyl group. As a result, aged forms of $T c \mathrm{AChE}$ and hBChE cannot be reactivated. 


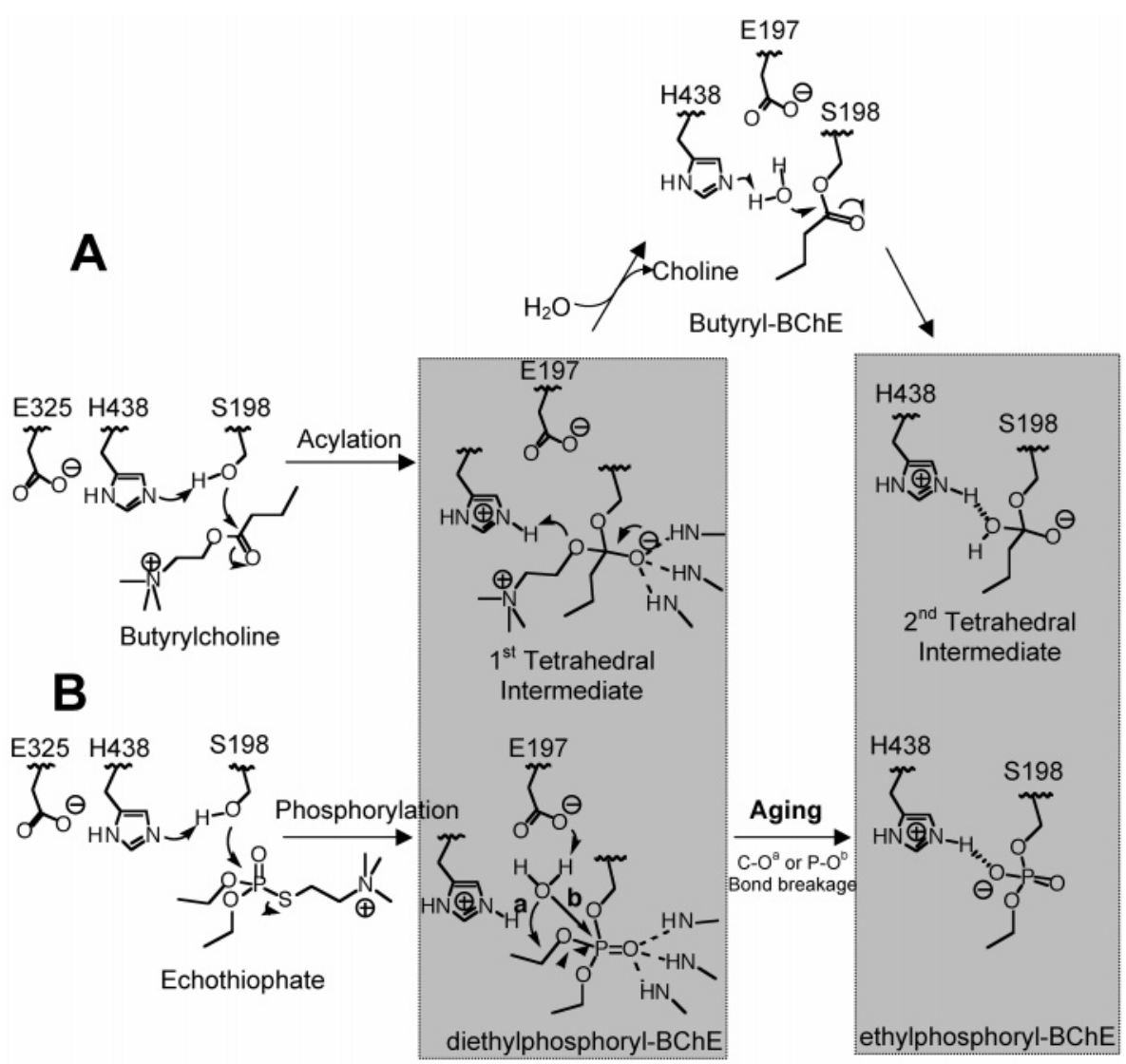

FIGURE 4: Catalytic hydrolysis mechanism of butyrylcholine by $\mathrm{hBChE}(\mathrm{A})$ and phosphorylation mechanism with the inhibitor echothiophate (B). The gray boxes indicate the similarities between the intermediates for the two mechanisms.

A second consequence is that a salt bridge forms between His $438 / 440$ and the oxyanion of the dealkylated OP, stabilizing the negative charge. Figure 3 panels $\mathrm{C}$ and $\mathrm{D}$ show that the salt bridge imposes identical orientations on His438/440 and on the dealkylated phosphyl group in echothiophateinhibited hBChE and in VX-inhibited TcAChE. This salt bridge raises the energy of activation necessary for the oxime-mediated enzyme reactivation. Moreover, the proton of the catalytic His, captured in the salt bridge, cannot be easily transferred to the serine as is required in the reactivation mechanism. In hBChE, formation of the salt bridge induces a slight rotation/shift of the phosphoryl moiety, aligning the negatively charged oxygen with His438 (Figure $3 \mathrm{~A}, \mathrm{C})$. In $T c \mathrm{AChE}$, formation of the salt bridge forces the catalytic His440 to return to its classical position where it is hydrogen-bonded to Glu327 (Figure 3B,D).

Glycerol in the Aged Structure Displaces Water Molecules Present in Nonaged $h B C h E$. There is a molecule of glycerol in the active site of the aged monoethylphosphoryl-hBChE (Figure 3C). The presence of glycerol is an artifact related to its use as a cryoprotectant during $\mathrm{X}$-ray diffraction experiments. Two hydroxyls of glycerol roughly superimpose with the location of water molecules 207 and 72 present before aging in the diethylphosphoryl-hBChE (not shown). These two hydroxyls are at hydrogen-bond distance with the negatively charged oxygen of the dealkylated phosphoryl. The third hydroxyl is within hydrogen-bond distance of Glu197. The glycerol binds to the aged active site because the negatively charged oxygen resulting from the dealkylation is a better hydrogen-bond acceptor than the equivalent phosphoester oxygen in the nonaged $\mathrm{hBChE}$. The butyrate-
$\mathrm{hBChE}$ complex also has an equivalent glycerol in the active site.

OP Conjugates as Analogues of Catalytic Intermediates. The mechanism of hydrolysis of butyrylcholine is illustrated in Figure 4A. The carboxylic ester undergoes a nucleophilic attack by the catalytic serine to form the first tetrahedral intermediate. This intermediate collapses to the butyrylenzyme intermediate with release of choline. Then a water molecule makes a nucleophilic attack on the butyryl-enzyme to form the second tetrahedral intermediate that collapses into the butyrate-enzyme complex. This butyrate-enzyme complex was seen in the crystal structure of native $\mathrm{hBChE}$ (PDB entry 1P0I).

The mechanism of inhibition and aging by echothiophate is illustrated in Figure 4B. The phosphoric acid thioester undergoes a nucleophilic attack by the catalytic serine to form a bipyramidal intermediate (not shown). This intermediate collapses to diethylphosphoryl- $\mathrm{hBChE}$ with release of the thiocholine. The diethylphosphoryl-hBChE intermediate (Figure 4B) is analogous to the first tetrahedral intermediate (Figure 4A) because both have the same tetrahedral geometry and the choline carboxyl ester function is analogous to one of the ethyl phosphoryl ester functions. One of the ethoxy groups points to the mouth of the gorge (Figure 4A). Similarly, in the nonaged $\mathrm{VX}-T c \mathrm{AChE}$ structure, the ethoxy group points toward the mouth of the gorge (Figure 4B). It was previously suggested that the orientation of the charged leaving group of cationic OPs is directed toward the mouth of the gorge (21). In the first tetrahedral intermediate, the choline head may similarly point toward Tyr332 of the peripheral anionic site. 


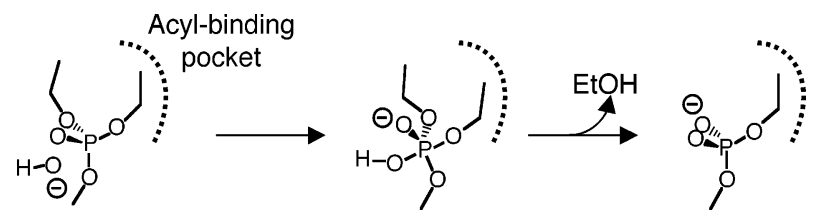

Figure 5: Aging mechanism by $\mathrm{P}-\mathrm{O}$ bond breaking.

A water molecule hydrogen-bonded to Glu197 was observed in the crystal structure of the diethylphosphoryl$\mathrm{hBChE}$. Although the first tetrahedral intermediate structure has never been directly observed, an equivalent water molecule is likely to be present in the same location. This water molecule is in the right position to attack the butyryl$\mathrm{hBChE}$ complex to form the second tetrahedral intermediate (22).

Both the second tetrahedral intermediate, that is, butyrate$\mathrm{hBChE}$, and the ethylphosphoryl $\mathrm{hBChE}$ were directly observed in crystal structures. These structures are analogous in the following ways. The isosteric butyryl and ethoxy chains interact with the acyl-binding pocket. The butyrate and ethoxy atoms superimpose. Both the carbonyl oxygen of the butyrate and the (oxy)phosphoryl oxygen are locked into the oxyanion hole. The anionic phosphoryl oxygen in the ethylphosphoryl-hBChE forms a salt bridge with the imidazolium, similar to the electrostatic interaction between the natural substrate and His438 in the second tetrahedral intermediate. In the ethylphosphoryl-hBChE the formal negative charge is distributed on the oxygen in the oxyanion hole and the oxygen in the salt bridge with His438. Both the oxyanion hole and the charged histidine stabilize the negative charge. In the second tetrahedral intermediate the negative charge is similarly stabilized by the oxyanion hole and the imidazolium. This dual stabilization may explain why butyrate $-\mathrm{hBChE}$ was found as a stable complex in the crystal.

\section{DISCUSSION}

Mechanism of Aging by Breaking $P-O$ Bond. The crystal structure of nonaged, echothiophate-inhibited $\mathrm{hBChE}$ shows one water molecule ideally positioned to catalyze aging by two mechanisms: by breaking the $\mathrm{C}-\mathrm{O}$ bond and/or by breaking the $\mathrm{P}-\mathrm{O}$ bond. An aging mechanism that involves breaking of the $\mathrm{P}-\mathrm{O}$ bond has never been reported. Breaking of a $\mathrm{P}-\mathrm{O}$ bond is chemically feasible as demonstrated by the fact that a $\mathrm{P}-\mathrm{O}$ bond between the $\mathrm{OP}$ and serine is broken during reactivation of OP-inhibited $\mathrm{BChE}$.

Our hypothesis requires a specific OP stereochemistry for aging by $\mathrm{P}-\mathrm{O}$ bond breaking. Common pesticides have no chiral phosphorus atom. Their active forms are usually dimethyl or diethyl phosphate. Therefore, pesticides always place an alkoxy group in the acyl-binding pocket, which is a requirement for $\mathrm{P}-\mathrm{O}$ bond breaking (nucleophilic substitution by in-line inversion). A bipyramidal intermediate is formed when the water molecule close to Glu197 attacks the phosphorus atom (Figure 5). The stereochemistry of the phosphorus atom imposes that the leaving group be on the opposite side of the attacking molecule of water, that is, in the acyl-binding pocket. As a consequence, OP pesticides are expected to age through $\mathrm{P}-\mathrm{O}$ bond breaking, in addition to aging through $\mathrm{C}-\mathrm{O}$ bond breaking.

By contrast, nerve agents have a chiral phosphorus atom. Their stereoisomers do not systematically fulfill the require- ment for $\mathrm{P}-\mathrm{O}$ bond breaking. A stereoisomer that places an alkyl group in the acyl-binding pocket cannot undergo $\mathrm{P}-\mathrm{O}$ bond breaking. For example, VX is a mixture of $\mathrm{P}(S)$ and $\mathrm{P}(R)$ stereoisomers. The $\mathrm{P}(S)$ stereoisomer reacts stereospecifically with $T c \mathrm{AChE}$, so that the methyl group of the moiety lies in the acyl-binding pocket, whereas the $\mathrm{P}(R)$ stereoisomer yields the ethoxy group in the acyl-binding pocket. The $\mathrm{P}(S)$ stereoisomer can age only by $\mathrm{C}-\mathrm{O}$ bond cleavage, whereas the $\mathrm{P}(R)$ stereoisomer can age only by breaking of the $\mathrm{P}-\mathrm{O}$ bond.

The acyl-binding pocket of $\mathrm{hBChE}$ can accommodate bigger substituents than TcAChE. Consequently, unlike TcAChE, hBChE does not exert stereoselectivity for OP bearing small substituents. This is the case for sarin (methyl and isopropyloxy groups) and VX (methyl and ethoxy groups) (23). hBChE can react with both stereoisomers of sarin or $\mathrm{VX}$, while $T c \mathrm{AChE}$ reacts preferentially with the stereoisomer that yields the smallest substituent in the acylbinding pocket. TcAChE does not present a potential leaving group in the acyl-binding pocket when it is inhibited by sarin or VX; therefore aging occurs exclusively by $\mathrm{C}-\mathrm{O}$ bond breaking. The $\mathrm{C}-\mathrm{O}$ bond breaking is always the favored aging mechanism for compounds with branched alkoxy groups, like soman, sarin, and DFP. The intermediate carbocation is not stabilized by neighboring carbons for nonbranched alkoxy groups such as ethoxy and methoxy. As a result the $\mathrm{C}-\mathrm{O}$ bond breaking is slower. We expect both $T c \mathrm{AChE}$ and $\mathrm{hBChE}$ to age by $\mathrm{P}-\mathrm{O}$ bond breaking as well as by $\mathrm{C}-\mathrm{O}$ bond breaking when the conjugate is a dimethoxy or diethoxy phosphate.

The $\mathrm{P}-\mathrm{O}$ bond breaking mechanism of aging is similar to the aging mechanism described for $T c \mathrm{AChE}$ and $\mathrm{hBChE}$ inhibited by $(1 S, 3 S)$-isomalathion $(24,25)$. $(1 S, 3 S)$-Isomalathion conjugates of $\mathrm{AChE}$ and $\mathrm{BChE}$ aged via an $\mathrm{S}_{\mathrm{N}} 2$ mechanism with loss of diethyl thiosuccinate, that is, by breaking of $\mathrm{P}-\mathrm{S}$ bond. The $\mathrm{P}-\mathrm{S}$ bond breakage is so favorable that the fast rate of aging was impossible to measure by conventional methods. It is expected that the rate of $\mathrm{P}-\mathrm{O}$ bond breaking is much slower because of the nature of the leaving group, thiols being better leaving groups than alcohols.

Regarding phosphoramidyl conjugates, NMR studies showed that tabun ages slowly through a mechanism involving the $\mathrm{P}-\mathrm{N}$ bond scission (26). It is possible that the $\mathrm{P}-\mathrm{N}$ bond scission occurs via an $\mathrm{S}_{\mathrm{N}} 2$ mechanism similar to the $\mathrm{P}-\mathrm{O}$ bond mechanism of aging we propose.

Role of Protonated Histidine in Aging by $\mathrm{P}-\mathrm{O}$ Bond Breaking. Because His438 is positively charged, it reinforces the electrophilicity of the phosphorus atom by withdrawing the electrons on the neighboring alkoxy oxygen. A second role of His 438 is to hold water molecule 101 by H-bonding, at the optimum location for the nucleophilic attack of the phosphorus atom. It is noteworthy that His 438 cannot participate in the activation of the water molecule because it cannot accept a second proton. The activation of water relies on Glu197.

The catalytic histidine cannot play these two roles when it is shifted like in the nonaged VX-inhibited TcAChE structure. In this case, His440 is away from the alkoxy oxygen so that it does not provide an electrostatic environment favorable to the $S_{N} 2$ mechanism. Since His 440 is unavailable, water molecule 1009 interacts only with Glu199. 
Unlike the position of His438, the position of His440 is unfavorable for $\mathrm{P}-\mathrm{O}$ bond breaking.

Evidence for Aging by $\mathrm{S}_{\mathrm{N}} 2$ Reaction? $\mathrm{pH}$ dependence studies were helpful to understand the mechanism of aging of soman-inhibited cholinesterase (27). The dealkylation rate of soman-inhibited huBChE is faster when both His 438 and Glu197 are ionized because the imidazolium ion stabilizes a negative charge on the ethoxy oxygen while the negatively charged glutamate stabilizes the carbocation intermediate. Hobbiger (28) showed earlier that the aging of diethylphosphoryl- bovine $\mathrm{AChE}$ is faster at $\mathrm{pH} 6.0$ than 8.0 and dependent on the protonation of a residue of $\mathrm{p} K_{\mathrm{a}}$ about 7.3, that is, the catalytic histidine. This $\mathrm{pH}$ dependence study supports that aging of diethylphosphoryl AChE occurs principally by dealkylation. However, it is not clear that the $\mathrm{pH}$ dependence of the $\mathrm{S}_{\mathrm{N}} 2$ reaction would be much different from that of the dealkylation, even in this $\mathrm{pH}$ range, because as described above, a protonated catalytic histidine also constitutes a driving force for the $\mathrm{S}_{\mathrm{N}} 2$ reaction. Besides, the $\mathrm{S}_{\mathrm{N}} 2$ rate may not be significantly enhanced by the higher concentration of hydroxyl ions at high $\mathrm{pH}$ because Glu197 repulses approaching anions. The rate of the $\mathrm{S}_{\mathrm{N}} 2$ reaction should be enhanced at low $\mathrm{pH}$ close to the $\mathrm{p} K_{\mathrm{a}}$ of Glu197, where Glu197 can act as a general base catalyst to activate water. Therefore a $\mathrm{pH}$-dependence study is not appropriate to discriminate between the two mechanisms.

A better method to provide evidence of the $\mathrm{S}_{\mathrm{N}} 2$ mechanism is to age in the presence of $\mathrm{H}_{2}{ }^{18} \mathrm{O}$ and then analyze the aged products by mass spectrometry. ${ }^{18} \mathrm{O}$ will be incorporated on the monoethylphosphoryl group only if aging results from the nucleophilic attack of $\mathrm{H}_{2}{ }^{18} \mathrm{O}$. Thus, there will be a mass difference of $+2 \mathrm{Da}$ between the $\mathrm{S}_{\mathrm{N}} 2$ product and the dealkylation product, that can be detected by mass spectrometry analysis of tryptic peptides. This experiment is underway.

Why BChE Ages Faster than AChE. Comparison of the rates of aging of $\mathrm{hBChE}$ and $T c \mathrm{AChE}$ in Table 1 shows that diethoxy and diisopropoxy conjugates of $\mathrm{hBChE}$ age about 4-fold faster than the conjugates with $T c \mathrm{AChE}$. A possible explanation for this difference is that His 438 in $\mathrm{hBChE}$ is always well positioned to carry out the dealkylation reaction, whereas His440 in $T c \mathrm{AChE}$ shifts to a position unfavorable for aging (10). The absence of a histidine shift in hBChE is supported by NMR studies. Viragh et al. (29) observed only one short strong hydrogen bond in aged $\mathrm{hBChE}$, between His438 and Glu325. In contrast, Massiah et al. (30) observed two short strong hydrogen bonds in aged-hAChE, one between His440 and Glu327 and one between His440 and Glu199. This suggests a conformational heterogeneity of the catalytic histidine in hAChE that does not exist in $\mathrm{hBChE}$.

In summary, the crystal structures of DFP-, somaninhibited $\mathrm{hBChE}$, and $T c \mathrm{AChE}$ show that the acyl-loop adapts its conformation to fit moderate-size substituents in the acyl-binding pocket. The crystal structures of aged and nonaged echothiophate-inhibited $\mathrm{hBChE}$ show the conformational stability of the catalytic histidine and the presence of a molecule of water able to promote aging by an $\mathrm{S}_{\mathrm{N}} 2$ mechanism involving $\mathrm{P}-\mathrm{O}$ bond cleavage.

\section{ACKNOWLEDGMENT}

We thank Larry M. Schopfer for helpful scientific discussions.

\section{REFERENCES}

1. Broomfield, C. A., Maxwell, D. M., Solana, R. P., Castro, C. A., Finger, A. V., and Lenz, D. E. (1991) Protection by butyrylcholinesterase against organophosphorus poisoning in nonhuman primates, J. Pharmacol. Exp. Ther. 259, 633-638.

2. Wolfe, A. D., Blick, D. W., Murphy, M. R., Miller, S. A., Gentry, M. K., Hartgraves, S. L., and Doctor, B. P. (1992) Use of cholinesterases as pretreatment drugs for the protection of rhesus monkeys against soman toxicity, Toxicol. Appl. Pharmacol. 117, 189-193.

3. Ashani, Y., and Pistinner, S. (2004) Estimation of the upper limit of human butyrylcholinesterase dose required for protection against organophosphates toxicity: a mathematically based toxicokinetic model, Toxicol. Sci. 77, 358-67.

4. Michel, H. O., Hackley, B. E., Jr., Berkowitz, L., List, G., Hackley, E. B., Gillilan, W., and Pankau, M. (1967) Aging and dealkylation of Soman (pinacolylmethylphosphonofluoridate)-inactivated eel cholinesterase, Arch. Biochem. Biophys. 121, 29-34.

5. Amitai, G., Moorad, D., Adani, R., and Doctor, B. P. (1998) Inhibition of acetylcholinesterase and butyrylcholinesterase by chlorpyrifos-oxon, Biochem. Pharmacol. 56, 293-299.

6. Skrinjaric-Spoljar, M., Simeon, V., and Reiner, E. (1973) Spontaneous Reactivation and Aging of dimethylphosphorylated Acetylcholinesterase and Cholinesterase, Biochim. Biophys. Acta 315, 363-369.

7. Kovarik, Z., Radic, Z., Berman, H. A., Simeon-Rudolf, V., Reiner, E., and Taylor, P. (2003) Acetylcholinesterase active centre and gorge conformations analysed by combinatorial mutations and enantiomeric phosphonates, Biochem. J. 373, 33-40.

8. Masson, P., Froment, M. T., Bartels, C. F., and Lockridge, O. (1997) Importance of aspartate-70 in organophosphate inhibition, oxime re- activation and aging of human butyrylcholinesterase, Biochem. J. 325, 53-61.

9. Millard, C. B., Kryger, G., Ordentlich, A., Greenblatt, H. M., Harel, M., Raves, M. L., Segall, Y., Barak, D., Shafferman, A., Silman, I., and Sussman, J. L. (1999) Crystal structures of aged phosphonylated acetylcholinesterase: nerve agent reaction products at the atomic level, Biochemistry 38, 7032-7039.

10. Millard, C. B., Koellner, G., Ordentlich, A., Shafferman, A., Silman, I., and Sussman, J. (1999) Reaction products of acetylcholinesterase and VX reveal a mobile histidine in the catalytic triad, J. Am. Chem. Soc. 121, 9883-9884.

11. Nachon, F., Nicolet, Y., Viguie, N., Masson, P., Fontecilla-Camps, J. C., and Lockridge, O. (2002) Engineering of a monomeric and low-glycosylated form of human butyrylcholinesterase: expression, purification, characterization and crystallization, Eur. J. Biochem. 269, 630-637.

12. Nicolet, Y., Lockridge, O., Masson, P., Fontecilla-Camps, J. C., and Nachon, F. (2003) Crystal structure of human butyrylcholinesterase and of its complexes with substrate and products, J. Biol. Chem. 278, 41141-7.

13. Otwinowski, Z. (1997) Processing of X-ray diffraction data collected in oscillation mode, Methods Enzymol. 276, 307-326.

14. Collaborative Computational Project Number 4. (1994) The CCP4 suite: Programs for Protein Crystallography, Acta Crystallogr. D 50, 760-763.

15. Vagin, A., and Teplyakov, A. (1997) MOLREP: an automated program for molecular replacement, J. Appl. Crystallogr. 30, $1022-1025$.

16. Murshudov, G. N. (1997) Refinement of macromolecular structures by the maximum-likelihood method, Acta Crystallogr. D 53, 24055.

17. Delano, W. L. (2002) The PyMOL Molecular Graphics System, DeLano Scientific, San Carlos, CA.

18. Jones, T. A., Zou, J. Y., Cowan, S. W., and Kjeldgaard. (1991) Improved methods for building protein models in electron density maps and the location of errors in these models, Acta Crystallogr. A 47, 110-9.

19. Viragh, C., Kovach, I. M., and Pannell, L. (1999) Small molecular products of dealkylation in soman-inhibited electric eel acetylcholinesterase, Biochemistry 38, 9557-9561.

20. Harris, L. W., Fleisher, J. H., Clark, J., and Cliff, W. J. (1966) Dealkylation and loss of capacity for reactivation of cholinesterase inhibited by sarin, Science 154, 404-7.

21. Hosea, N. A., Radic, Z., Tsigelny, I., Berman, H. A., Quinn, D. M., and Taylor, P. (1996) Aspartate 74 as a primary determinant in acetylcholinesterase governing specificity to cationic organophosphonates, Biochemistry 35, 10995-11004. 
22. Nair, H. K., Seravalli, J., Arbuckle, T., and Quinn, D. M. (1994) Molecular recognition in acetylcholinesterase catalysis: freeenergy correlations for substrate turnover and inhibition by trifluoro ketone transition-state analogues, Biochemistry 33, 85668576.

23. Hosea, N. A., Berman, H. A., and Taylor, P. (1995) Specificity and orientation of trigonal carboxyl esters and tetrahedral alkylphosphonyl esters in cholinesterases, Biochemistry 34, 1152811536.

24. Doorn, J. A., Talley, T. T., Thompson, C. M., and Richardson, R. J. (2001) Probing the active sites of butyrylcholinesterase and cholesterol esterase with isomalathion: conserved stereoselective inactivation of serine hydrolases structurally related to acetylcholinesterase, Chem. Res. Toxicol. 14, 807-813.

25. Doorn, J. A., Thompson, C. M., Christner, R. B., and Richardson, R. J. (2003) Stereoselective inactivation of Torpedo californica acetylcholinesterase by isomalathion: inhibitory reactions with $(1 R)$ - and $(1 S)$-isomers proceed by different mechanisms, Chem. Res. Toxicol. 16, 958-65.

26. Barak, D., Ordentlich, A., Kaplan, D., Barak, R., Mizrahi, D., Kronman, C., Segall, Y., Velan, B., and Shafferman, A. (2000) Evidence for $\mathrm{P}-\mathrm{N}$ bond scission in phosphoroamidate nerve agent adducts of human acetylcholinesterase, Biochemistry 39, 11561161.

27. Saxena, A., Viragh, C., Frazier, D. S., Kovach, I. M., Maxwell, D. M., Lockridge, O., and Doctor, B. P. (1998) The pH dependence of dealkylation in soman-inhibited cholinesterases and their mutants: further evidence for a push-pull mechanism, Biochemistry 37, 15086-15096.

28. Hobbiger, F. (1956) Chemical reactivation of phosphorylated human and bovine true cholinesterases, Br. J. Pharmacol. 11, 295-303.

29. Viragh, C., Harris, T. K., Reddy, P. M., Massiah, M. A., Mildvan, A. S., and Kovach, I. M. (2000) NMR evidence for a short, strong hydrogen bond at the active site of a cholinesterase, Biochemistry 39, 16200-16205.

30. Massiah, M. A., Viragh, C., Reddy, P. M., Kovach, I. M., Johnson, J., Rosenberry, T. L., and Mildvan, A. S. (2001) Short, strong hydrogen bonds at the active site of human acetylcholinesterase: proton NMR studies, Biochemistry 40, 5682-5690.

31. Mason, H. J., Waine, E., Stevenson, A., and Wilson, H. K. (1993) Aging and spontaneous reactivation of human plasma cholinesterase activity after inhibition by organophosphorus pesticides, Hum. Exp. Toxicol. 12, 497-503.

32. Masson, P., Fortier, P. L., Albaret, C., Froment, M. T., Bartels, C. F., and Lockridge, O. (1997) Aging of di-isopropyl-phosphorylated human butyrylcholinesterase, Biochem. J. 327, 601607.

33. Worek, F., Eyer, P., and Szinicz, L. (1998) Inhibition, reactivation and aging kinetics of cyclohexylmethylphosphonofluoridateinhibited human cholinesterases, Arch. Toxicol. 72, 580-587.

34. Main, A. R. (1979) Mode of action of anticholinesterases, Pharmacol. Ther. 6, 579-628.

35. Clothier, B., Johnson, M. K., and Reiner, E. (1981) Interaction of some trialkyl phosphorothiolates with acetylcholinesterase. Characterization of inhibition, aging and reactivation, Biochim. Biophys. Acta 660, 306-316.

36. Berry, W. K., and Davies, D. R. (1966) Factors influencing the rate of "aging" of a series of alkyl methylphosphonyl-acetylcholinesterases, Biochem. J. 100, 572-576.

37. Shafferman, A., Ordentlich, A., Barak, D., Stein, D., Ariel, N., and Velan, B. (1996) Aging of phosphylated human acetylcholinesterase: Catalytic processes mediated by aromatic and polar residues of the active centre, Biochem. J. 318, 833-840.

38. Brunger, A. T. (1992) Free $R$ value: a novel statistical quantity for assessing the accuracy of crystal structures, Nature 355 , $472-475$.

BI048238D 